\title{
Self-assembly and Sensor Response of Photosynthetic Reaction Centers on
}

$$
\text { Screen-printed Electrodes. }
$$

\author{
Vijayender Bhalla ${ }^{+}$, Valter Zazubovich ${ }^{*}$
}

\author{
Department of Physics, Concordia University, 7141 Sherbrooke St. West, Montreal,Quebec \\ H4B 1R6, Canada
}

\section{Abstract}

Photosynthetic reaction centers were immobilized onto gold screen-printed electrodes (Au-SPE) using a self-assembled monolayer (SAM) of mercaptopropionic acid (MPA) which was deliberately defective in order to achieve effective mediator transfer to the electrodes. The pure Photosystem II (PS II) cores from spinach immobilize onto the electrodes very efficiently but fair

badly in terms of photocurrent response (measured using duroquinone as the redox mediator).

5 The cruder preparation of PS II known as BBY particles performs significantly better under the same experimental conditions and shows a photocurrent response of 20 to $35 \mathrm{nA}$ (depending on

7 preparation) per screen-printed electrode surface $\left(12.5 \mathrm{~mm}^{2}\right)$. The data was corroborated using AFM, showing that in the case of BBY particles a defective biolayer is indeed formed, with grooves spanning the whole thickness of the layer enhancing the possibility of mass transfer to the electrodes and enabling biosensing. In comparison, the PS II core layer showed ultra-dense organization, with additional formation of aggregates on top of the single protein layer, thus

| * Corresponding author; phone (1-514) 848-2424 \#5050; fax (1-514) 848-2828; e-mail: .1 vzazubov@alcor.concordia.ca 'Present Address: Biosensors Laboratory, Institute of Microbial Technology, Sector 39A Chandigarh India 160036; vkbhalla@imtech.res.in 
22 blocking mediator access to the electrodes and/or binding sites. The defective monolayer

23 biosensor with BBY particles was successfully applied for the detection of photosynthesis

24 inhibitors, demonstrating that the inhibitor binding site remained accessible to both the inhibitor

25 and the external redox mediator. Biosensing was demonstrated using picric acid and atrazine.

26 The detection limits were $1.15 \mathrm{nM}$ for atrazine and $157 \mathrm{nM}$ for picric acid.

27

28 Keywords: Photosynthetic reaction centers; Gold screen printed electrode; Self-assembled

29 monolayer; Herbicide detection; Atrazine; Picric acid.

| * Corresponding author; phone (1-514) 848-2424 \#5050; fax (1-514) 848-2828; e-mail: $\quad .2$ vzazubov@alcor.concordia.ca 'Present Address: Biosensors Laboratory, Institute of Microbial Technology, Sector 39A Chandigarh India 160036; vkbhalla@ imtech.res.in 


\section{Introduction}

Biosensing, one of the many possible practical applications of biomolecules, requires the

32 controlled immobilization of biomolecules in close contact with electrochemical transducers.

33 Self-assembled monolayers (SAM) provide a unique tunable platform since the thickness of the

34 organic layers and surface properties are adjustable to suit different sensing applications [1]. The

35 organic molecules forming SAM feature different anchor groups that can be used to attach

36 various classes of biomolecules [2]. They also provide some level of control over packing

37 density at the surface [3]. Generally, use of SAMs allows the biochemical reaction to proceed in

38 a more controlled manner thus enhancing biosensing parameters [4].

39 One common approach towards selective herbicide detection is based on the use of

40 antibodies [5]. However, generation of antibodies against small molecules is tedious and time

41 consuming process, and requires animal models to raise the antibodies. Antibody-based detection

42 may also face problems due to cross-reactivity of antibodies with similar compounds [6].

43 Antibodies are fairly large objects, especially compared to small herbicide molecules;

44 additionally, blocking in immunoassays is a complex problem that has to be addressed before

45 any useful data is obtained. Summarizing, there is an apparent need to continue looking for

46 alternative approaches, one of them being to employ the natural photosynthesis machinery for

47 detection purposes. Photosynthetic biosensors are capable of detecting a broad spectrum of

48 herbicides and have generated a lot of interest as an alternative to antibody-based biosensing.

49 Whole photosynthetic organisms [7], as well as bacterial reaction centers [8], have been used for

50 this purpose. The most common version makes use of Photosystem II (PS II), the photosystem

51 that is also responsible for water splitting and oxygen production. The initial reports on PS II-

52 based herbicide biosensors employed electrochemical flow cells with PS II in suspension [9] or 
53 immobilized with the help of different substances [10-12]. Clark electrode-based setup that

54 monitors changes in oxygen evolution activity of the PS II was used in [13]. More recent reports

55 again focused on amperometric detection, with the photosynthetic material entrapped in gel

56 matrices on top of the electrodes [14-17]. Combining electrochemical and optical detection has

57 been recently reported in [18]. In PS II the illumination induces charge separation, with electron

58 eventually traveling to mobile plastoquinone $\mathrm{Q}_{\mathrm{B}}$. In vivo the latter accepts two electrons (and

59 two protons), transforms to quinol and carries the electrons away. The mechanism of inhibition

60 of PS II by herbicides in vivo involves herbicide molecules attaching to the $\mathrm{Q}_{\mathrm{B}}$ binding site and

61 preventing plastoquinone from binding. The exposure of the PS II-based biosensor to the

62 inhibitor results in a decrease of the photoinduced current in an electrical circuit containing the

63 photosynthetic reaction centers, because the mediator (replacing plastoquinone) cannot bind to

64 the $\mathrm{Q}_{B}$ site.

65 Although entrapping photosynthetic materials in gels allows for reasonable accessibility

66 due to the porous nature of the matrix, it has some inherent limitations. The main limitations are

67 due to swelling or contraction of gels with time, poor adhesion to the electrodes, and the stress of

68 fluid movement that may lead to washing off of certain materials trapped in the matrix. The

69 diffusion coefficient of mediators and herbicides in different gels, polymers and other matrices is

70 also a limiting factor. Covalent immobilization using BSA-glutaraldehyde has been found to be

71 better than other schemes as it is a simple one-step procedure based on cross-linking of amines

72 that results in a very stable matrix system on top of the electrodes $[16,18]$. Another procedure,

73 resulting in preservation of photosynthetic activity for somewhat longer time involves

74 immobilization using poly(vinylalcohol) bearing styrylpyridinium groups (PVA-SbQ) [19]. 
The immobilization of photosynthetic materials in a monolayer fashion is quite

76 challenging and has been of interest for various applications, including bioelectrocatalytic fuel

77 cells [20]. The immobilization of PS II with the help of SAMs has been carried out using

78 Histidine-tagged PS II that attaches to nickel on the NTA (nitrilotriacetic acid) SAM [21]. This

79 technology requires genetic engineering to introduce the histidine tag into the PS II. Moreover,

80 Ni-NTA-terminated SAM preparation on gold electrodes involves a multistep protocol. Thus,

81 although this method leads to immobilization of the reaction centers in a uniformly-oriented

82 fashion, the mass transfer to the electrodes becomes limited due to multiple layering steps

83 effectively insulating the electrodes. Maly et al. [22] studied this topic and suggested deliberately

84 creating defect structures using BSA in the PS II sensing biolayer to achieve increased mass

85 transfer efficiency to the electrodes while working with NTA-SAM as the linker molecule.

86 However, this approach could lead to decreased current due to the co-immobilization of BSA on

87 the sensing surface.

88 In the present report we suggest a simpler approach that makes use of carboxylic acid

89 anchoring groups of MPA SAM on gold screen-printed electrode (Au SPE) surface to bind

90 native PS II reaction centers or membrane fragments for unique biointerface development. The

91 MPA films are known to exhibit many pinhole defects [23]. We utilize these pinhole defects to

92 achieve effective mass transfer to the electrodes.

93

\section{2. Experimental}

95 All chemicals were purchased from Sigma-Aldrich (USA). Organic baby spinach leaves

96 were purchased from the local food suppliers. Buffer compositions were as follows: 
97 Homogenizing buffer: $20 \mathrm{mM}$ MES (pH 6.0), $15 \mathrm{mM} \mathrm{NaC1}, 5 \mathrm{mM} \mathrm{CaCl}_{2}$. Measuring buffer: 15

$98 \mathrm{mM}$ 2-(N-morpholino) ethanesulfonic acid (MES), pH 6.5, $0.5 \mathrm{M}$ mannitol, $0.1 \mathrm{M} \mathrm{NaCl}, 5 \mathrm{mM}$

$99 \mathrm{MgCl}_{2}$, and $5 \times 10^{-5} \mathrm{M}$ chloramphenicol (supplemented with $0.2 \mathrm{mM} \mathrm{DQ}$ ).

100

$101 \quad 2.1$ Isolation of PS II-containing particles

102

The BBY particles [24] refer to PS II-enriched membrane fragments. The BBY particles

103 are mostly devoid of Photosystem I, but still retain the oxygen evolving capacity and some lipid

104 membranes within which the hydrophobic mediator can travel and reach its binding site. Both

105 core and peripheral antenna complexes of PS II are retained. BBY particles were obtained after

106 treatment of thylakoids with Triton X-100 at a final concentration of $25 \mathrm{mg}$ per $\mathrm{mg} \mathrm{Chl}$ and

107 repeated centrifugation for $25 \mathrm{~min}$ at $40,000 \mathrm{~g}$ ) in homogenizing buffer. The chlorophyll

108 concentration for all purposes was determined by the method of Arnon [25].

109 The PS II core particles were prepared similarly to [26]. These particles constitute the

110 minimal PS II preparation still retaining the oxygen-evolving capacity, and consist of the PS II

111 reaction center, as well as CP43 and CP47 core antenna complexes. Note that in this case the

112 photosynthetic protein is encased into the detergent micelle and the original thylakoid membrane

113 is not retained.

114

\section{$115 \quad 2.2$ Surface preparation procedures}

116 A $2 \mathrm{mM}$ MPA solution prepared in a 75/25\% ethanol/water mixture (vol/vol) was used

117 for the formation of SAMs. Gold surfaces were incubated for 1 hour, in the dark, and then rinsed

118 with ethanol. After that sonication in ethanol/water was carried out for 5 minutes in order to

119 remove physisorbed thiols from the gold surface. The surfaces were further washed with 
120 deionized water and dried with nitrogen. For AFM investigations (see Sections 2.4 and 3.2), the

121 SAM was formed not on a screen-printed electrode but on gold substrate (100 nm thickness)

122 prepared by electron beam evaporation on a silicon dioxide chip with a $5 \mathrm{~nm}$ titanium stick layer.

123 The gold surface was cleaned in a piranha solution (mixture of $3: 1$ of $\mathrm{H}_{2} \mathrm{SO}_{4}$ and $\mathrm{H}_{2} \mathrm{O}_{2}$ ) for 30

$124 \mathrm{~min}$ before deposition of SAM.

125 After SAM formation, the electrodes were treated for 10 minutes with a mixture of NHS

126 - N-hydroxysuccinimide (0.05 M) and EDC - Ethyl-Dimethyl-aminopropyl Carbodiimide (0.2

127 M) solutions in distilled deionized water. As a zero-degree cross-linking agent EDC does not

128 introduce any additional chemical groups between the conjugating molecules. EDC reacts with

129 carboxyl groups of the MPA SAM, forming an amine-reactive $o$-acylisourea intermediate. This

130 intermediate in turn can react with amines of the photosynthetic material forming amide bonds

131 and releasing isourea by-product [27]. A further incubation (6 hours at $4{ }^{\circ} \mathrm{C}$ in the dark) was

132 carried out with the PS II particle suspension. The electrodes were carefully washed in MES

133 buffer and dried with nitrogen after each incubation step.

\section{$135 \quad 2.3$ Photo-electrochemical Measurements}

136 Gold screen-printed electrodes were purchased from DropSens Inc. (model DRP-220.

137 The electrode assembly consists of a gold working electrode (area $12.57 \mathrm{~mm}^{2}$ ) and a gold 138 counter electrode. The reference electrodes and electrical contacts were made of silver and 139 screen printed on a ceramic substrate $3.4 \times 1 \times 0.05 \mathrm{~cm}$ (length $\mathrm{x}$ width $\mathrm{x}$ thickness). All potential 140 values are reported with respect to silver pseudo reference electrode. The electrochemical 
141 response of the electrodes with and without immobilized material was investigated using the CHI 142 630C electrochemical workstation.

143 The schematics of the biosensor are presented in Figure 1. For amperometric detection of

144 photosynthesis inhibitors the $I-t$ curves were measured at room temperature, with $50 \mu l$ droplets 145 of the measuring buffer placed onto the working area covering the three electrodes. Duroquinone 146 (DQ; $0.2 \mathrm{mM}$ ) was used as a mediator in these experiments and, respectively, the working 147 electrode was polarized at $0.62 \mathrm{~V}[16,28]$. Quinones are used as mediators in PS II-based 148 herbicide biosensors due to their similarity to plastoquinone which binds to the $\mathrm{Q}_{\mathrm{B}}$ site in vivo. 149 DQ in particular was employed as a mediator also in $[13,14,17]$. Other mediators used in PS-II 150 based biosensors include 2,5-dichlorobenzoquinone [11], 2,6-dichlorophenolindophenol [18] and 151 ferricyanide [10,16,28]. Ferricyanide, although providing the largest photocurrents, is clearly not 152 specific for the $\mathrm{Q}_{\mathrm{B}}$ site [16,28]. A $7 \mathrm{~mW}$ laser diode with $675 \mathrm{~nm}$ wavelength (near the peak of 153 the PS II $\mathrm{Q}_{\mathrm{y}}$ absorption band) was used for illumination. In the absence of light only small dark 154 current is registered. Illuminating the sensor leads to a significant increase in the detected current 155 which is due to light-induced charge separation in the PS II. Turning the light off results in the 156 return of the current to the pre-illumination levels (See also sections 3.1 and 3.3). Addition of 157 photosynthesis inhibitors results in a decrease of the magnitude of the photo-induced current 158 peak. [Suggested location of Fig.1)]

\section{$160 \quad 2.4$ AFM Characterization}

161 AFM studies were performed in order to assess the quality of SAM formation and 162 photosynthetic material immobilization. The AFM images were obtained in air, while operating 163 in tapping mode, using a Digital Instruments Multimode AFM with a standard sharpened $\mathrm{Si}_{3} \mathrm{~N}_{4}$ 
164 tip (cantilever resonant frequency was $300 \mathrm{kHz}$ ). The images were collected with high resolution 165 (512 points per line) at a scan rate of $1-2 \mathrm{~Hz}$. Raw images were only processed for background 166 removal (flattening) using the AFM manufacturer's software.

\section{Results and Discussion}

\subsection{Electrochemical and photo-electrochemical characterization}

The gold screen printed electrodes were used for thiol films formation. Not many reports

171 have previously focused on thiol-Au films formation on screen printed electrodes; notable

172 exceptions include [29,30]. Our goal was to form a non-insulating SAM (that would allow free

173 movement of the mediator to the electrode surface) using short chain alkanethiols. It is well

174 known that as the chain length decreases, the degree of order of SAM's decreases as well, 175 together with the packing density and surface coverage [31]. The well-known redox curve of 176 potassium ferrocyanide on gold electrode surface is presented in Figure 2 (solid curve). This 177 curve can be compared with the curve measured for the electrodes covered with MPA SAM 178 (dashed curve). The response is clearly decreased. On the other hand, the features of the cyclic 179 voltammograms demonstrate that the thiol SAM is not perfectly insulating as the redox reaction 180 of ferrocyanide is still accessible [32]. For comparison, almost no current is detected in case of 181 highly-ordered SAM [33]. [Suggested location of Figure 2]

182 The proper immobilization of PS II core particles on MPA SAM was confirmed by 183 observing the redox reaction for the various cofactors naturally present in the PS II structure. The 184 cyclic voltammetry (CV) technique was used to characterize development of proper biointerface 185 on SPE. The CV scans of immobilized core particles were obtained in MES buffer $\mathrm{pH} 6.5$ and 186 showed a reversible peak and a non-reversible peak when investigated using screen printed $\mathrm{Au}$ 
187 electrodes with a silver pseudo-reference electrode (Supplemental Information, Fig. S1). A 188 reversible peak at redox midpoint potential of $-0.086 \mathrm{~V}$ can be ascribed to the (native) quinones $189\left(\mathrm{Q}^{-} \mathrm{Q}^{-}\right)$, and the irreversible peak at $\sim 0.22 \mathrm{~V}$ can be ascribed to the tetramanganese $\left(\mathrm{Mn}_{4}\right)$ cluster 190 which shows that it is intact and accessible to the electrochemical reaction as described earlier 191 [34]. The fact that this reaction is observed indicates close contact between PS II and the 192 electrodes due to the short chain length of the SAM material.

193 Figure 3 compares the photocurrent signal measured as the reoxidation of the 194 duroquinone (DQ) mediator at $0.62 \mathrm{~V}$ for the immobilized BBY sample in the cases of BSA195 glutaraldehyde matrix system (A) and SAM (B), solid curves. The photocurrent signal is higher 196 in case of SAM as compared to matrix-based immobilization for the same area of the electrode. 197 As can be seen in the picture there is a significant difference in the sensor's response in these two 198 cases. The difference arises mainly from the re-oxidation rate of the reduced mediator. In the 199 case of immobilization of PS II on the SAM layer the mediator can access the electrode surface 200 more easily. In the case of the matrix system, on the other hand, the speed of this process is 201 limited by the diffusion rate of the mediator in the gel matrix and the reoxidation process takes 202 longer [35]. The dotted curve in the frame B is an example of the signal in the presence of 203 photosynthesis inhibitor. Surprisingly, the photocurrent signal in the case of PS II cores was 1.00 $204 \pm 0.75 \mathrm{nA}$ only, significantly smaller than for BBY particles. [Suggested location of Figure 3]

205 Concerning the biosensor stability, in the case of BBY particles it took about 24 hours for 206 the photocurrent signal to be reduced by half (see Supplemental Information, Fig. S2). The 207 photocurrent did not show significant decay in the first 2 hours, most probably due to the 208 stabilization effect of the natural lipid membrane environment. 
The photocurrent generation properties observed using duroquinone as a mediator were

212 significantly different for BBY particles and PS II core preparations. AFM investigation allowed

213 us to shed more light on the possible reasons of these differences. The AFM imaging was

214 conducted using flat evaporated gold surface rather than the surface of the screen-printed

215 electrodes. Thus, we managed to elucidate the fine details of SAM formation and photosynthetic

216 material binding which could otherwise be partially masked by the higher surface roughness of

217 the screen-printed electrodes. According to SEM images presented at manufacturer's website

(http://www.dropsens.com), the surface of the screen-printed electrodes used in this work features

granules of the size of $\sim 2 \mu \mathrm{m}$, not very suitable for detailed AFM investigation. On the other

hand, this is at least an order of magnitude larger than features described below. Thus, we

believe that the details of SAM formation and PS II immobilization do not differ drastically

between evaporated gold surface and screen-printed electrode. The quality of the SAM and of

the bio-layer can be characterized by root mean square (RMS) roughness. Table 1 summarizes

224 the RMS roughness values for bare surface as well as the surfaces after various modifications.

225 The RMS roughness for the bare gold surface was $1.05 \pm 0.1 \mathrm{~nm}$. The deposition of the SAM

226 led to a small increase in the surface roughness to $1.85 \pm 0.25 \mathrm{~nm}$. Figure 4 shows the AFM

227 images of the bare gold surface and the MPA SAM on the gold surface. The topology of a nicely

228 formed SAM almost perfectly follows the gold layer's corrugation, although defects in SAM

229 such as cracks or patches would contribute to an increase in RMS roughness [36]. The

230 immobilization of the photosynthetic complexes leads to a significant increase of the roughness.

231 The RMS roughness increases to $21.9 \mathrm{~nm}$ with the immobilization of PS II core particles and to

$2328.67 \mathrm{~nm}$ for BBY membranes. The larger roughness in case of the pure PS II core sample is most 
233 likely due to cluster formation as illustrated in the schematic accompanying Figure 5A. For BBY

234 membranes we do not observe aggregate formation upon immobilization. The thickness of the

235 layer is approximately $10 \mathrm{~nm}$, in agreement with values previously reported for these particles

236 using AFM imaging [22]. The AFM image of the immobilized PS II core particles on the MPA

237 SAM (Figure 5A) show some repeatable features with the size (in the plane of the layer) of

238 approximately $50 \mathrm{~nm}$, as well as some objects of larger size, $100 \mathrm{~nm}$ and beyond. The height of

239 the former features is approximately $10 \mathrm{~nm}$. These must be the clusters of PS II core particles

240 since individual PS II core dimers have been reported to have much smaller size, namely $20.6 \mathrm{x}$

$24113.1 \mathrm{~nm}$, with thickness varying from $6.0 \mathrm{~nm}$ on the periphery of the complex to $9.1 \mathrm{~nm}$ in the

242 RC region [37]. In [37] the thickness of the detergent layer around the hydrophobic surface of

243 the protein was estimated at $1.6 \mathrm{~nm}$ only. Incidentally, in an earlier report the AFM images

244 contained some 40-60 nm features for histidine-modified PS II immobilized on nickel-

245 nitriloacetic acid (Ni-NTA) SAM [22]. Another report described smaller features whose size was

246 consistent with that of the PS II dimers [38]. The high purity and homogeneity of this protein

247 preparation allows immobilizing it in a very dense manner thus most likely completely blocking

248 mediator access to the electrodes or the $\mathrm{Q}_{\mathrm{B}}$ sites. (Although the PS II core samples were

249 detergent-solubilized, one also cannot exclude a possibility that hydrophobicity of the

250 complexes, isolated from natural membranes, contributes to their aggregation.) This result is in

251 accordance with those by other researchers who found higher protein densities to interfere with

252 biosensor assays mainly by interfering with the diffusion of the analyte to the enzyme or by

253 hindering electron transfer to the electrode surface [39]. In addition, higher protein loading on

254 the surface, particularly for enzymes, has been shown to neutralize active sites or alter the

255 morphology of the enzyme through mutual interactions [40]. Thus, the poor performance of this 
preparation, in our case, was ascribed to the higher protein density, in agreement with the results

257 of other researchers who found high protein densities to be a limiting factor in the performance

258 of biosensors [41-43]. It is also possible that detergent micelle, as opposed to native thylakoid

259 membrane present in BBY particles, is preventing the access of the mediator to the $\mathrm{Q}_{\mathrm{B}}$ site.

260 In the case of BBY particles the membrane fragments successfully immobilize on the

261 surface but not in an ultra-dense manner. The image in Figure 5B shows heterogeneous features.

262 It has both areas (marked with a square) with uniform immobilization of relatively small

263 membrane fragments, as well as regions (outside of the square) with large membrane fragments

264 on top of the gold surface and possibly on top of each other. The presence of the natural

265 membrane environment in case of BBY particles likely allows the hydrophobic mediator to gain

266 better access to the $\mathrm{Q}_{\mathrm{B}}$ binding site. The presence of nanogaps in the film allows the reduced

267 mediator in solution to gain better access to the electrode surface as shown in schematic

268 accompanying Figure 5B. The smallest particles present in the AFM image in Figure 5B appear

269 to be approximately $25-30 \mathrm{~nm}$, consistent with the size of the dimeric PS II supercomplexes

270 containing peripheral antenna [37]. Figure 6 directly compares the cross-section features (along

271 the lines present in Figure 5 A and B, respectively) for the two sample preparations. In case of

272 the PS II cores the features repeat with the period of approximately $100 \mathrm{~nm}$, with occasional

273 larger aggregate formation. In case of BBY sample the grooves are observed in the biolayer,

274 spanning the total thickness of the biolayer i.e. $\sim 10 \mathrm{~nm}$. Thus in the latter case there is ample

275 opportunity for mediator to gain access to the electrode surface. [Suggested location for Figures

$2764,5,6]$

277

$278 \quad 3.3$ Detection of photosynthesis inhibitors 
Herbicides inhibit photosynthesis by interrupting electron transfer at the quinone-

280 reducing site of PS II. In vivo herbicides compete with the plastoquinone for its $\mathrm{Q}_{\mathrm{B}}$ binding site

281 on the D1 protein, thus leading to disruption of electron transfer from $\mathrm{Q}_{\mathrm{A}}$ to $\mathrm{Q}_{\mathrm{B}}$ and further along

282 the electron transport chain. In our experiments the herbicide binding to the $\mathrm{Q}_{\mathrm{B}}$ site did not allow

283 the mediator (DQ) to accept electrons from the site and hence the process of electron transfer

284 from PS II to the mediator and further to the electrode was stalled. The detection was based on

285 the decrease in photocurrent in the presence of herbicides (see Frame B of Figure 3).

286 Reference photocurrent was first obtained without the addition of herbicides. A

287 preconditioning phase of about 10 minutes was required before the photocurrent from a fresh

288 biosensor became stable. A droplet $(50 \mu \mathrm{l})$ of measuring buffer containing the mediator was

289 allowed to spread over the electrodes covered with immobilized PS II and the photocurrent

290 generated from the biosensor was measured for illumination time of 10 sec after 10 min of

291 incubation. The biosensor was then subjected to successive droplets containing increasing

292 concentrations of the herbicide and the light-induced current was measured, again after 10 min of

293 incubation. In between applying different herbicide concentrations the sensor surface was

294 washed with excess of measuring buffer (including DQ) to remove the herbicide. Each

295 measurement was recorded three times at the same concentration of the analyte (using fresh

296 droplets) to check for reproducibility.

297 The data for different analytes was plotted as residual activity versus concentration (on a

298 logarithmic scale), Figure 7. The residual activity is the activity of the biosensor in percent after

299 addition of the inhibitor; it is equal to the ratio of photocurrents in the presence and in the

300 absence of the inhibitor. Experimental data were fitted to a logistic equation describing a 301 sigmoidal binding curve. 


$$
R=\min +\frac{M a x-M i n}{1+(x / I C 50)^{H}}
$$

304 Here Max is the maximal activity before adding any analyte and Min is the minimum residual 305 activity, when sensor is saturated by the inhibitor; $H$ is the Hill slope, and $\mathrm{x}$ is the inhibitor concentration. The IC50 is the point midway between top and bottom of the sigmoidal curve.

307 The assumption behind the use of this curve is that the mediator and the inhibitor bind 308 competitively to one and the same site on the PS II. The limit of detection, LOD, was calculated 309 as

$$
L O D=I C 50\left(\frac{2.6 \sigma}{M a x-M i n-2.6 \sigma}\right)^{1 / H}
$$

311 see [28]. The factor of 2.6 corresponds to $99 \%$ confidence interval. Picric acid can be classified

312 as nitrophenolic herbicide according to its chemical structure and has been employed in research

313 on the feasibility of the PS II-based biosensors for explosives detection [28]. It has been

314 previously described to be an inhibitor of PS II in photosynthetic electron transport [44]. The

315 curve shifts towards higher concentrations for the picric acid in comparison to atrazine,

316 indicating a lower degree of picric acid binding to the $\mathrm{Q}_{\mathrm{B}}$ site. The fit parameters are presented

317 in Table 2. The $I C_{50}$ for picric acid is 15 times higher as compared to atrazine which signifies a

318 lower affinity of picric acid for the $\mathrm{Q}_{\mathrm{B}}$ binding site in comparison to triazine-type herbicides. The

319 developed assay showed an excellent dynamic response range between $1 \mathrm{nM}$ to $1 \mu \mathrm{M}$ for

320 detection for atrazine and LOD is $1.15 \mathrm{nM}$ indicating its potential application for environmental 321 analysis. In repeated experiments, the reproducibility (coefficient of variation) of the sensor for $322 \mathrm{n}=3$ measurements was $\sim 5 \%$, for $10 \mathrm{nM}$ atrazine concentration. The LOD of different atrazine 323 sensors reported in the literature are summarized in Table 3. The limit of detection of $1.15 \mathrm{nM}$ 
324 for atrazine is significantly lower than the Maximum Residue Level (MRL) (50 $\mu \mathrm{g} \mathrm{L}^{-1}$ or 232

$325 \mathrm{nM}$ ) established by EU (European Union) and close the MRLs for drinking water of each

326 individual pesticide at $0.1 \mu \mathrm{gL}^{-1}$ and the total amount of pesticides at $0.5 \mu \mathrm{g} \mathrm{L}-1(2.32 \mathrm{nM})$.

327 [Suggested location of Figure 7] For picric acid the sensor shows a relatively poor LOD of 157

$328 \mathrm{nM}$ which is mostly attributable to high $\sigma$. The LOD of $\sim 25 \mathrm{nM}$ has been reported for BSA

329 glutaraldehyde gel-immobilized PS II picric acid biosensor in [28]. The luminescence quenching

330 method yields LOD of $2 \mu \mathrm{M}$ [45]; employing the fluorescence emission of hexaphenysilole-

331 chitosan film the LOD of $\sim 21 \mathrm{nM}$ can be achieved [46]. It is important to point out that just like

332 most reported PS II-based herbicide biosensors [12,16,28], the one reported in this work is not

333 capable of distinguishing between different inhibitors without a priori knowledge of either the

334 nature of an inhibitor or the concentration. Thus, in its present form the biosensor is most

335 suitable for non-selective early-warning type applications. However, the use of genetically

336 modified PS II promises to allow better selectivity [18].

337 The main advantage of using SAM as compared to a matrix system is that due to smaller

338 biomolecule-to-electrode distance and to the absence of matrix which slows down the diffusion

339 of both analyte and the mediator, the equilibration and response times as well as the recovery

340 times are decreased, leading to lower illumination time being necessary. The peak response at

341 complete inhibition is near zero. It is possible to completely restore the signal by washing the

342 sensor with measuring buffer. The regeneration of the biosensor after experiments was almost

$343100 \%$ effective, in agreement with the results of [16]. It is also possible to reuse this sensor after

344 storage at $4^{\circ} \mathrm{C}$ within several hours, although within 24 hours the current drops substantially.

345 Longer-time storage of prepared sensors without loss of activity is possible at $-80^{\circ} \mathrm{C}$. 


\section{Conclusions}

This paper investigates a method for covalent immobilization of photosynthetic reaction 349 centers on top of a defective self-assembled monolayer that allows mediator to access the surface 350 of the electrodes easily. The photocurrent generation properties in case of BBY particles were 351 compared to results obtained with BSA-glutaraldehyde matrix based immobilization and they 352 show faster rise and decay of the photocurrent upon switching illumination on and off, and better 353 signal to noise ratio. The pure preparations of Photosystem II cores (with no lipids) from spinach 354 leaves immobilize very nicely on the electrode surface but fair badly in terms of photocurrent 355 generation properties. The AFM investigations helped us to better understand some of the 356 obtained results as we see that BBY particles organize themselves into a layer structure on top of 357 the SAM leaving certain free spaces. The PS II core preparation in turn shows a very dense 358 organization with aggregate formation that leaves no space for mediator to access the electrodes.

359 The action of photosynthesis inhibitors in reducing photo-induced current was demonstrated for 360 atrazine and picric acid. The obtained detection limits were $1.15 \mathrm{nM}$ and $157 \mathrm{nM}$, respectively.

362 Acknowledgments:

363 Authors are thankful to Dr. Rolf Schmidt, facility manager, Concordia University, for his help in 364 acquisition of AFM images and to Dr. C. Raman Suri (Pesticide Biosensors group) of IMTECH 365 Chandigarh, for fruitful discussions. The funding has been provided by NSERC under Strategic 366 Grants Program, Safety and Security. We acknowledge Defense R\&D Canada, RCMP, CBSA 367 and CATSA as supporting organizations. 


\section{References}

371 1. Z. Dai, H. Ju, Phys. Chem. Chem. Phys. 3 (2001) 3769-3773.

372 2. N.K. Chaki, K. Vijayamohanan, Biosens. Bioelectron. 17 (2002) 1-12.

373 3. S. Ferretti, S. Paynter, D.A. Russell, K.E. Sapsford, D.J. Richardson, Trends Anal.

$374 \quad$ Chem. 19 (2000) 530-540.

375 4. F. Frederix, K. Bonroy, W. Laureyn, G. Reekmans, A. Campitelli, W. Dehaen, G. Maes, $376 \quad$ Langmuir 19 (2003) 4351-4357.

377 5. C.R. Suri, R. Boro, Y. Nangia, S. Gandhi, P. Sharma, N. Wangoo, K. Rajesh, G.S.

378 Shekhawat, Trends Anal. Chem. 28 (2009) 29-39.

379 6. B. Byrne, E. Stack, N. Gilmartin, R.O. Kennedy, Sensors, 9 (2009) 4407-4445.

380 7. I. Shitanda, S. Takamatsu, K. Watanabe, M. Itagaki, Electrochim. Acta. 54 (2009) 49333814936.

382 8. H. Peters, C.S. Dannert, R D. Schmid. Mater. Sci. Eng. C 4 (1997) 227-232.

383 9. S. Lemieux, R. Carpentier, J. Photochem. Photobiol. B 2 (1988) 221-231.

384 10. S. Lemieux, R. Carpentier, Photochem. Photobiol. 48 (1988) 115-121.

385 11. C. Loranger, R. Carpentier, Biotechnol. Bioeng. 44 (1994) 178-183.

386 12. D. Laberge, J. Chartrand, R. Rouillon, R. Carpentier, Env. Toxicol. Chem. 18 (1999) $387 \quad 2851-2858$.

388 13. M. Koblízek, J. Masojídek, J. Komenda, T. Kucera, R. Pilloton, A. K. Mattoo, M. T. 389 Giardi, Biotech. Bioeng. 60 (1998) 664-669.

390 14. F. Bettazzi, L. Laschi, M. Mascini, Anal. Chim. Acta 589 (2007) 14-21.

391 15. E. Touloupakis, L. Giannoudi, S.A. Piletsky, L. Guzzella, F. Pozzoni, M.T. Giardi, 392 Biosens. Bioelectron. 20 (2005) 1984-1992. 
16. M. Koblízek, J. Maly, J. Masojídek, J. Komenda, T. Kucera, M.T. Giardi, A.K. Mattoo, R. 394 Pilloton, Biotechnol. Bioeng. 78 (2002) 110-116.

395 17. A. Tibuzzi, G. Pezzotti, T. Lavecchia, G. Rea, M. T. Giardi, Sens. Transducers 88 (2008) 9. 396 18. K. Buonasera, G. Pezzotti, V. Scognamiglio, A. Tibuzzi, M.T. Giardi, J. Agric. Food Chem. $397 \quad 58(2010) 5982-5990$.

398 19. E.V. Piletskaya, S.A. Piletsky, T.A. Sergeyeva, A.V. El'skaya, A. A. Sozinov, J.L. Marty and 399 R.Rouillon, Anal. Chim. Acta 391 (1999) 1-7.

400 20. K.B. Lam, E.F. Irwin, K.E. Healy and L. Lin, Sens. Act. B Chem. 117 (2006) 480-487.

401 21. N. Terasaki, M. Iwai, N. Yamamoto, T. Hiraga, S. Yamada, Y. Inoue, Thin Solid Films 516 402 (2008) 2553-2557.

403 22. J. Maly, J. Krejci, M. Ilie, L. Jakubka, J. Masojidek, R. Pilloton, K. Sameh, P. Steffan 404 Z. Stryhal, M. Sugiura. Anal. Bioanal. Chem. 381 (2005) 1558-1567.

405 23. S. Campuzano, M. Pedrero, C. Montemayor, E. Fatas, J.M. Pingarron, J. Electroanal. Chem. $406586(2006) 112-121$.

407 24. D.A. Berthold, G.T. Babcock, C.F. Yocum, FEBS Lett. 134 (1981) 231.

408 25. D.I. Arnon, Plant Physiol. 24 (1949) 1-15.

409 26. P.J. van Leeuwen, M.C. Nieveen, E.J. van de Meent, J.P. Dekker, H.J. Gorkom, Photosyn. $410 \quad$ Res. 28 (1991) 149-153.

411 27. T. Hermanson, In: Bioconjugate Techniques, Academic Press, San Diego, CA, 1996, pp. $412 \quad 139-140$.

413 28. V. Bhalla, X. Zhao, V. Zazubovich, J. Electoanal. Chem. 657 (2011) 84-90.

414 29. J. Shen, C.C. Liu, Sens. Actuators B: Chem. 120 (2007) 417-425.

415 30. O.A. Loaiza, S. Campuzano, M. Pedrero, J.M. Pingarron, Electroanal. 20 (2008) 1397-1405. 
416 31. C.D. Bain, E.B. Troughton, Y.Y. Tao, J. Evall, G.M. Whitesides, R.G. Nuzzo, J. Am. Chem. 417 Soc. $111(1989)$ 321-335.

418 32. V. Anandan, R. Gangadharan, G. Zhang, Sensors 9 (2009) 1295-1305.

419 33. V. Bhalla, S. Carrara, C. Stagni, B. Samorì, Thin Solid Films 518 (2010) 3360-3366.

420 34. Alcantara, B. Munge, Z. Pendon, H.A. Frank, J.F. Rusling, J. Am. Chem. Soc. 128 (2006)

$421 \quad 14930-14937$.

422 35. J. Maly, A. Masci, J. Masojidek, M Sugiura, R. Pilloton, Anal. Letts. 37 (2004) 1645-1656.

423 36. S. Carrara, V. Bhalla, C. Stagni, B. Samorì, Surf. Sci. 603 (2009) 75-77.

424 37. E. J. Boekma, B. Hankamer, D. Bold, J. Kruip, J. Nield, A. F. Boonstra, J. Barber, M.

425 Rogner, Proc. Natl. Acad. Sci. USA 92 (1995) 175-179.

426 38. A. Morrin, A. Guzman, A. J. Killard, J. M. Pingarron, M. R. Smyth, Biosens. Bioelectron. 18 $427 \quad$ (2003) 715-720.

428 39. M. Vittadello, M.Y. Gorbunov, D.T. Mastrogiovanni, L.S. Wielunski, E.L. Garfunkel, F.

429 Guerrero, D. Kirilovsky, M. Sugiura, A.W. Rutherford, A. Safari, P.G. Falkowski,

430 ChemSusChem 3 (2010) 474-475.

431 40. R. Ganapathy, S. Manolache, M. Sarmadi, W. J. Simonsick Jr., F. Denes, J. Appl. Polym. 432 Sci. $78(2000)$ 1783-1796.

433 41. Z. Naal, J.H. Park, S. Bernhard, J.P. Shapleigh, C.A Batt, H.D. Abrua, Anal. Chem. 74 434 (2002) 140-148.

435 42. R.D. Richins, A. Mulchandi, W. Chen. Biotechnol. Bioeng. 69 (2000) 591-596.

436 43. M. Darder, E. Casero, F. Pariente, E. Lorenzo, Anal Chem. 72 (2000) 3784-3792.

437 44. W. Oettmeier, K. Masson, Eur. J. Biochem. 122 (1982) 163-167.

438 45. J. Lu, Z. Zhang, Anal. Chim. Acta. 318 (1996) 175. 
439 46. G. He, H. Peng, T. Liu, M. Yang, Y. Zhang, Y. Fang, J. Mater. Chem. 19 (2009) 7347.

440 47. S. Hleli, C., Martelet, A. Abdelghani, N. Burais, N., Jaffrezic-Renault, Sens. Actuators B 113

441 (2006) 711-717.

442 48. E. Valera, J. Ramón-Azcón, F.-J. Sanchez, M.-P. Marco, Á. Rodríguez, Sens. Actuators B

$443 \quad 134(2008)$ 95-103

444 49. J. Přibyl, M. Hepel, J. Halamek, P. Skladal, Sens. Actuators B 91 (2003) 333-341.

445 50. C. Steegborn, P. Skladal, Biosens. Bioelectron. 12 (1997) 19-27.

446 51. C.R. Suri, J. Kaur, S. Gandhi, G.S. Shekhawat, Nanotechnology 19 (2008) 235502.

447 52. R. Wilson, M.H. Barker, D.J. Schiffrin, R Abuknesha, Biosens. Bioelectron. 12 (1997) 277448286.

449 53. J. Kaur, R.C.Boro N. Wangoo, K. Rajesh, C.R. Suri, Anal. Chim. Acta. 607 (2008) 92-98. 


\section{Figure Captions}

453 Figure 1. Schematics of the biosensor employed in this work. PS II-containing particles are 454 immobilized on top of a defective monolayer of MPA on a gold electrode. Light induces charge 455 separation in PS II and after several steps the electrons are accepted at the $\mathrm{Q}_{\mathrm{B}}$ binding site by a 456 non-native quinone (duroquinone, DQ) The reduced DQ leaves the $\mathrm{Q}_{\mathrm{B}}$ site and is eventually 457 oxidized at the gold SPE and the photo-induced current is detected. Introduction of 458 photosynthesis inhibitors interrupts this chain of events and the photoinduced current is reduced.

Figure 2. CV scans obtained using $30 \mathrm{mM}$ ferrocyanide in measuring buffer (no DQ) for bare 461 Au-SPE (solid curve) and after MPA SAM formation (dashed curve). The scan rate was $50 \mathrm{mV}$ $462 \sec ^{-1}$.

464 Figure 3. Comparison of photocurrent signal from BBY particle biosensor in case of (A) BSA465 glutaraldehyde matrix immobilization and (B) immobilization onto a self-assembled MPA 466 monolayer in the absence of inhibitors, solid curves. The illumination time is 20 see for (A) and $46710 \mathrm{sec}$ for (B). The dotted curve in frame B is the photocurrent peak in the presence of an 468 inhibitor, superimposed on the figure for illustrative purposes.

Figure 4. AFM images. Square side is $5.0 \mu \mathrm{m}^{z}$. a) Bare gold surface and b) Gold surface after formation of MPA SAM. In the image (b), the brighter regions correspond to the condensed

472 thiol islands (liquid or solid phase), and the darker regions correspond to the dilute phase (bare 473 Au surface). 
475 Figure 5. AFM images of PS II particles immobilized on a MPA monolayer; square sidze 2.5 $\mu \mathrm{m}^{z}$. a) PS II cores from spinach. Immobilized particles show cluster formation thus blocking 477 mediator access to the electrode surface; b) BBY particles: immobilized particles as well as 478 access sites to electrodes are visible. The RMS roughness in the highlighted square region is 4.16 $479 \mathrm{~nm}$. Below the AFM images the respective schematic drawings of the biolayers are presented.

481 Figure 6. Cross-sectional views along the lines present in Figure 4. a) PS II core particles are 482 located right next to each other and some aggregates are formed. b) Highly disordered situation 483 in case of BBY membranes, with grooves clearly spanning the whole thickness of the biolayer.

485 Figure 7. Calibration curves for the decrease of photocurrent upon addition of picric acid (open 486 circles) and atrazine (solid circles) in the presence of $0.2 \mathrm{mM} \mathrm{DQ}$. The experimental points were 487 fitted using Eq.1. 
Table 1. RMS roughness for various samples/surfaces

\begin{tabular}{|c|c|}
\hline Sample & $\begin{array}{c}\text { RMS roughness in } \mathrm{nm} \\
\text { for } 2.5 \mu \mathrm{m}^{2} \text { square }\end{array}$ \\
\hline Bare evaporated gold & $1.05 \pm 0.1$ \\
\hline MPA-SAM & $1.85 \pm 0.25$ \\
\hline PS II cores & 21.9 \\
\hline BBY & 8.67 \\
\hline
\end{tabular}

Table 2. Fitting parameters (Eqs. 1 and 2) for picric acid and atrazine

\begin{tabular}{|c|c|c|c|c|c|c|c|}
\hline & $\begin{array}{c}\text { Min } \\
(\%)\end{array}$ & $\begin{array}{c}\text { Max } \\
(\%)\end{array}$ & $\begin{array}{c}\text { EC50 } \\
(\mathrm{nM})\end{array}$ & $\sigma$ & $\begin{array}{c}\text { Hill } \\
\text { slope }\end{array}$ & $\mathrm{R}^{2}$ & $\begin{array}{c}\text { LOD } \\
(\mathrm{nM})\end{array}$ \\
\hline atrazine & 7.6 & 100.0 & 49 & 1.57 & 0.82 & 0.9984 & 1.15 \\
\hline $\begin{array}{c}\text { picric } \\
\text { acid }\end{array}$ & 10.4 & 100.0 & 784 & 4.83 & 1.13 & 0.9936 & 157.5 \\
\hline
\end{tabular}


Table 3. Examples of atrazine biosensors

\begin{tabular}{|c|c|c|}
\hline Ref. & Methodology & LOD \\
\hline [47] & Impedimetric, label-free immunosensor & $\begin{array}{c}20 \mathrm{ng} / \mathrm{ml} \\
93 \mathrm{nM}\end{array}$ \\
\hline [48] & Impedimetric, label-free immunosensor & $\begin{array}{c}8.34 \pm 1.37 \mathrm{ng} / \mathrm{ml} \\
39 \mathrm{nM}\end{array}$ \\
\hline [49] & Piezoelectric, label-free immunosensor & $\begin{array}{c}1.5 \mathrm{ng} / \mathrm{ml} \text { (direct) } \\
7 \mathrm{nM} / \\
0.025 \mathrm{ng} / \mathrm{mL} \text { (competitive) } \\
0.11 \mathrm{nM}\end{array}$ \\
\hline$[50]$ & Piezoelectric & $\begin{array}{c}0.1 \mu \mathrm{g} / 1 \\
0.46 \mathrm{nM}\end{array}$ \\
\hline [51] & $\begin{array}{l}\text { Nanomechanical } \\
\text { Cantilever based }\end{array}$ & $\mathrm{pM}$ \\
\hline$[52]$ & $\begin{array}{c}\text { Electrochemiluminescence flow injection } \\
\text { immunoassay }\end{array}$ & $\begin{array}{c}0.1 \mathrm{ppb} \\
6 \mathrm{nM}\end{array}$ \\
\hline [53] & Direct hapten coatin microtiter plates & $\begin{array}{l}20 \mathrm{ng} / \mathrm{L} \\
0.09 \mathrm{nM}\end{array}$ \\
\hline This work & PSII biosensor & $\begin{array}{c}247 \mathrm{ng} / \mathrm{L} \\
1.15 \mathrm{nM}\end{array}$ \\
\hline
\end{tabular}




\section{Figure}

Click here to download high resolution image

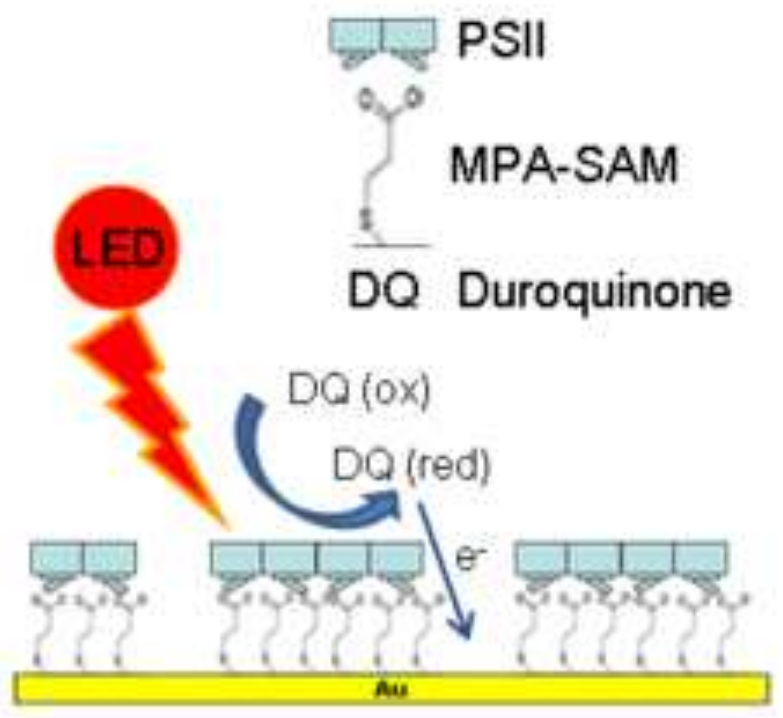


Figure
Click here to download high resolution image

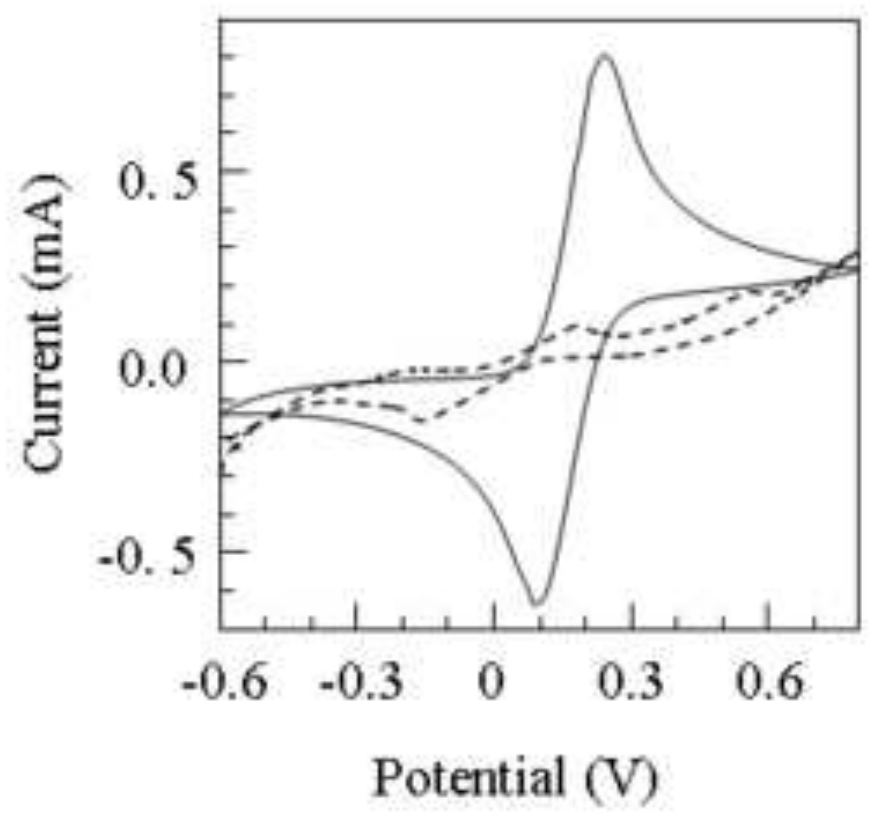

Figure 2 


\section{Figure}

Click here to download high resolution image

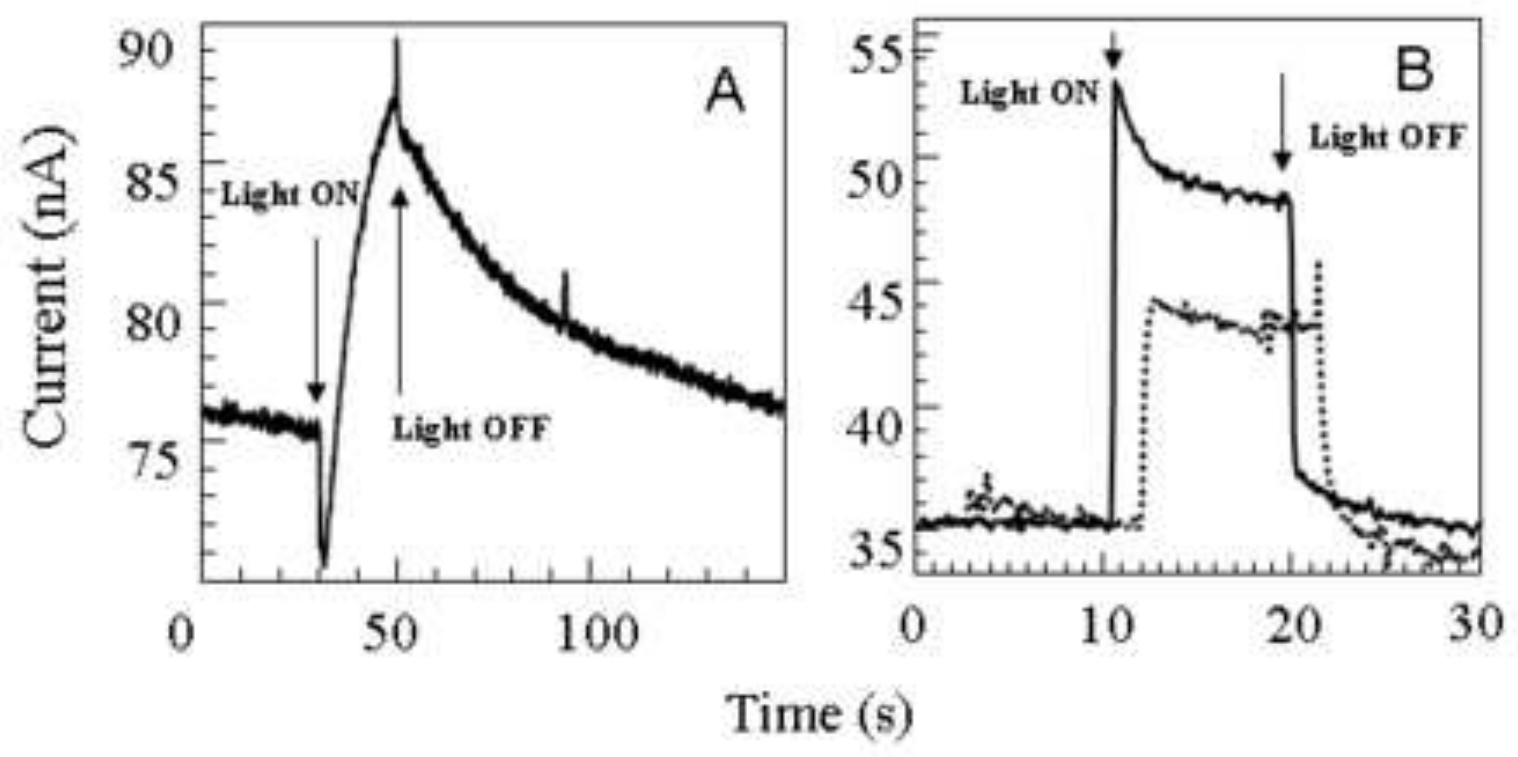

Figure 3 
Click here to download high resolution image
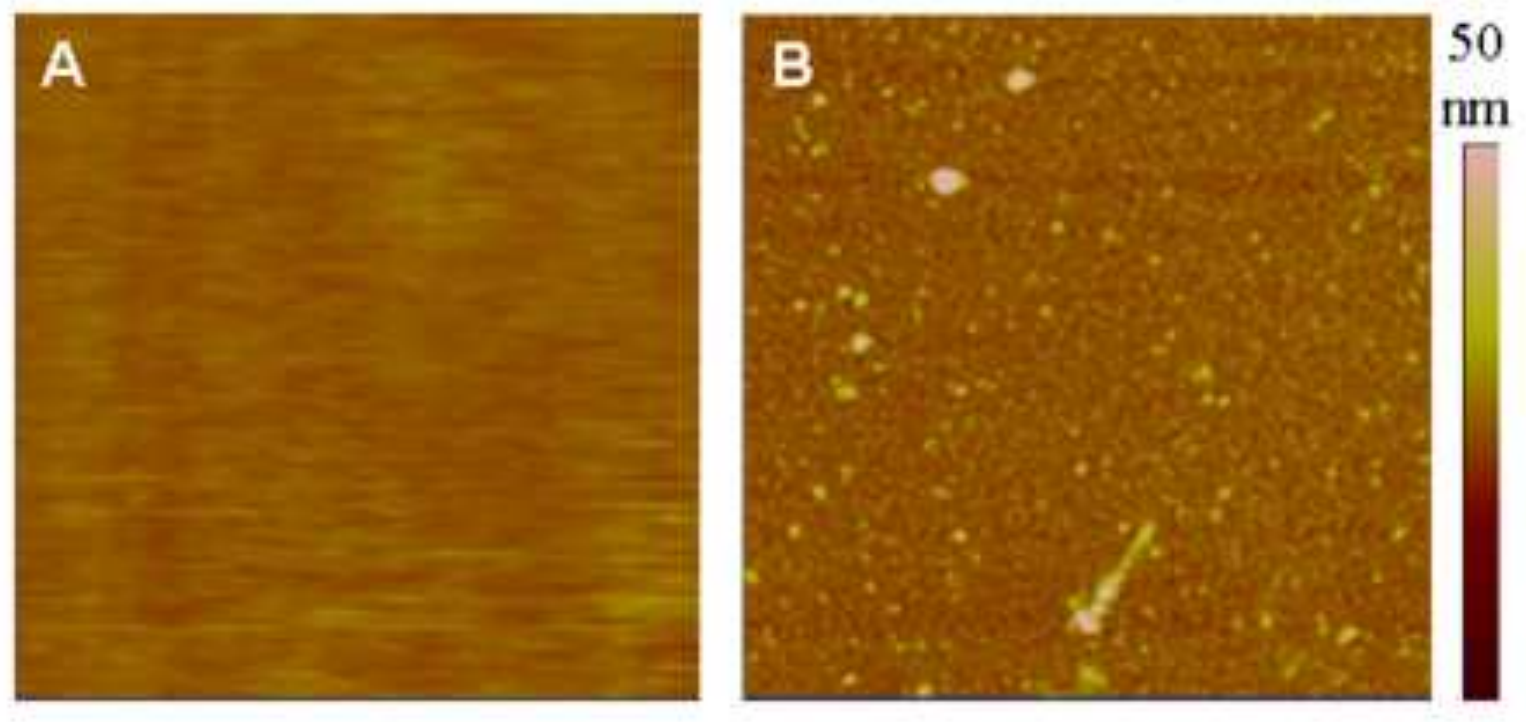

Figure 4 

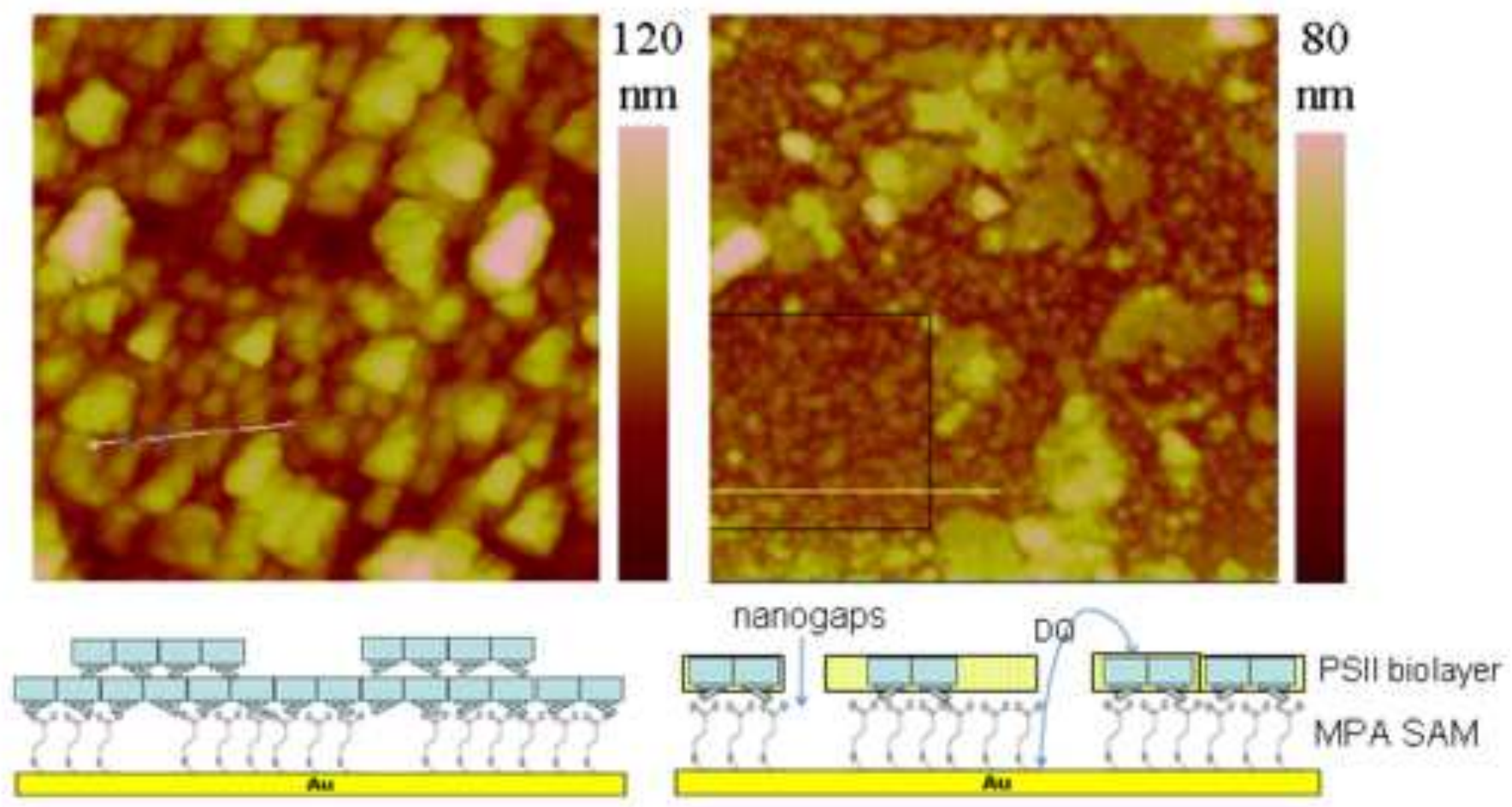
Figure
Click here to download high resolution image
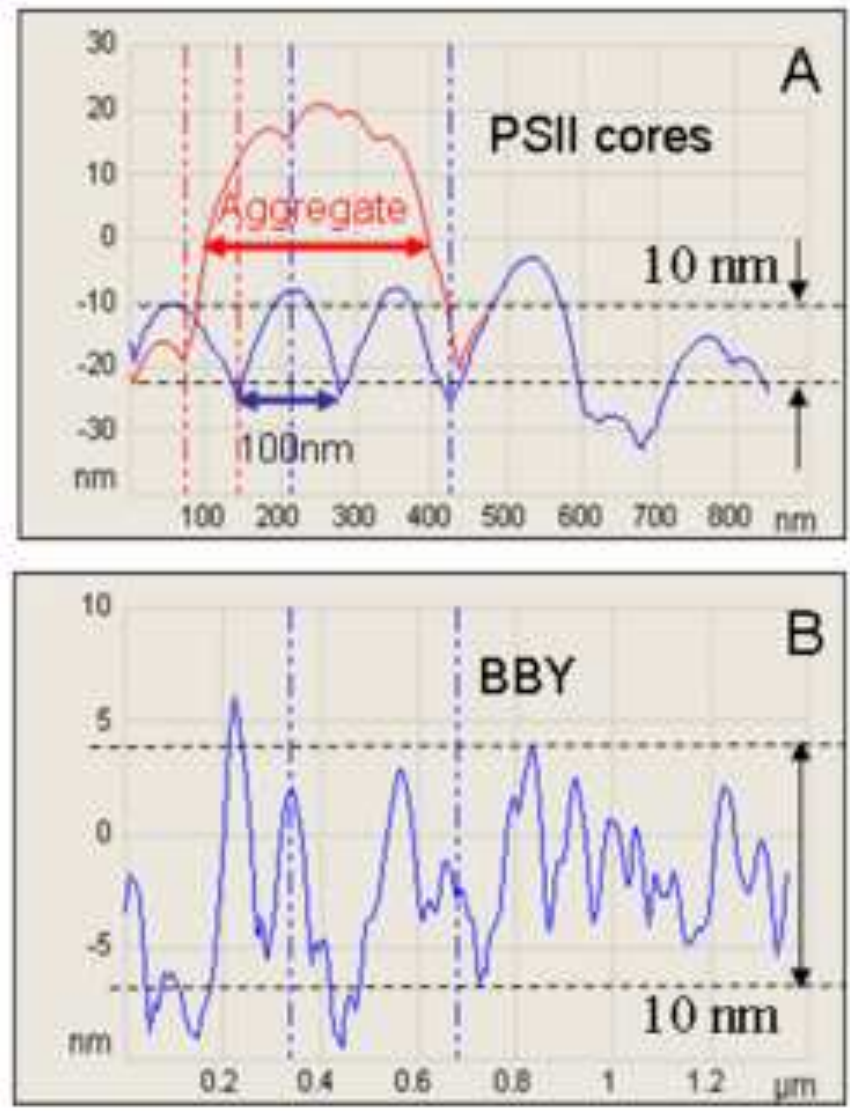

Figure 6 


\section{Figure}

Click here to download high resolution image

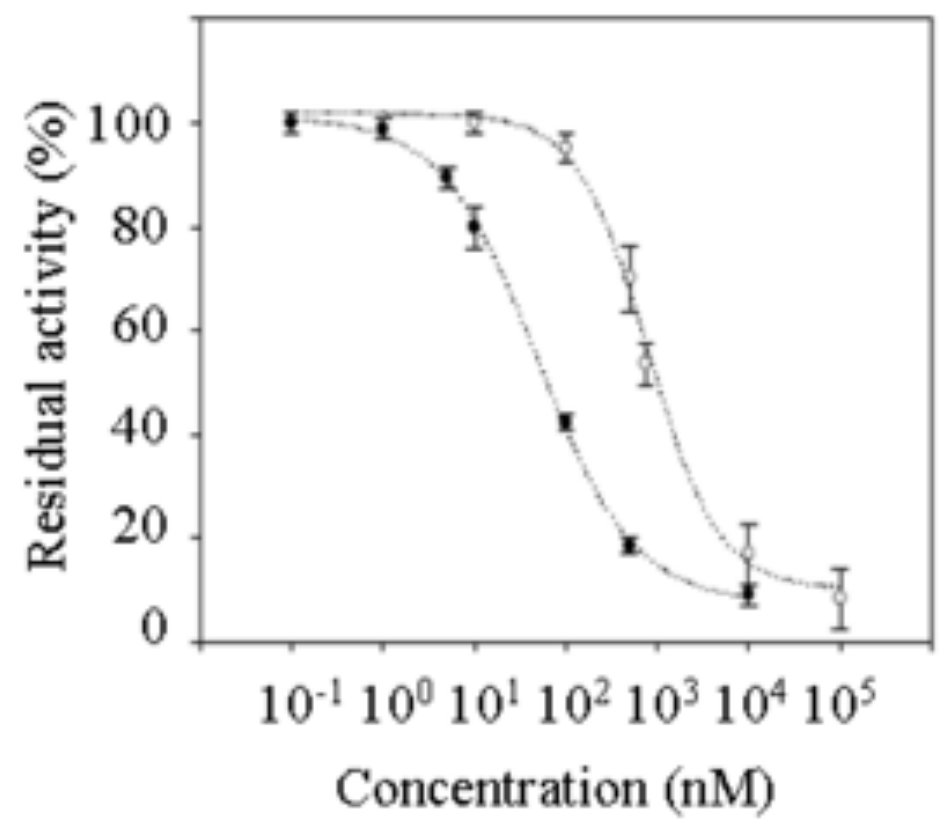

Figure 7 
Electronic Supplementary Material (online publication only)
Click here to download Electronic Supplementary Material (c

Click here to download Electronic Supplementary Material (online publication only): Supplementary Information.doc 